\title{
THE SHAPE OF MID-IR PAH BANDS IN THE UNIVERSE
}

\author{
O. Berné ${ }^{1}$, P. Pilleri ${ }^{2,3}$ and C. Joblin ${ }^{2,3}$
}

\begin{abstract}
A large number of galactic and extragalactic sources exhibit a mid-IR spectrum that is dominated by PAH emission. Looking at these spectra in more details reveals a strong variability in the shape/position of the observed features, depending on the observed source, or even the region within a source. In this article, we present the results of an analysis that has allowed us to decompose these spectra into components having a physical meaning. Most, if not all PAH dominated mid-IR spectra of HII regions, PDRs, protoplanetary disks, galaxies etc. can be fitted efficiently using a combination of these components. The results of these fits provide further insight in the composition of the emitting material and the local physical conditions. In the frame of the future IR space missions (JWST, SPICA), this approach can be very useful to probe the physical conditions in distant galaxies.
\end{abstract}

\section{Introduction}

The ubiquitous mid-IR emission bands, widely observed in the spectra of dusty astrophysical sources (from protoplanetary disks to starburst galaxies), are commonly attributed to the emission of polycyclic aromatic hydrocarbons (PAHs). However, because these bands are due to nearest neighbor vibrations of the $\mathrm{C}-\mathrm{C}$ or $\mathrm{C}-\mathrm{H}$ bonds, they are not specific to individual PAH species. Because essentially any PAH molecule will carry these bonds, the emission bands associated to vibrations are not discriminant to specific PAH molecules, thus preventing from any individual identification. However, these bands do carry some information, in particular their position in wavelength and their relative intensity are known to vary strongly depending on the chemical evolution of their carriers that is driven

\footnotetext{
${ }^{1}$ Leiden Observatory, Leiden University, Niels Bohrweg 2, 2333 CA Leiden, The Netherlands

${ }^{2}$ Université de Toulouse, UPS, CESR, 9 avenue du Colonel Roche, 31028 Toulouse Cedex 4, France

3 CNRS, UMR 5187, 31028 Toulouse, France
} 

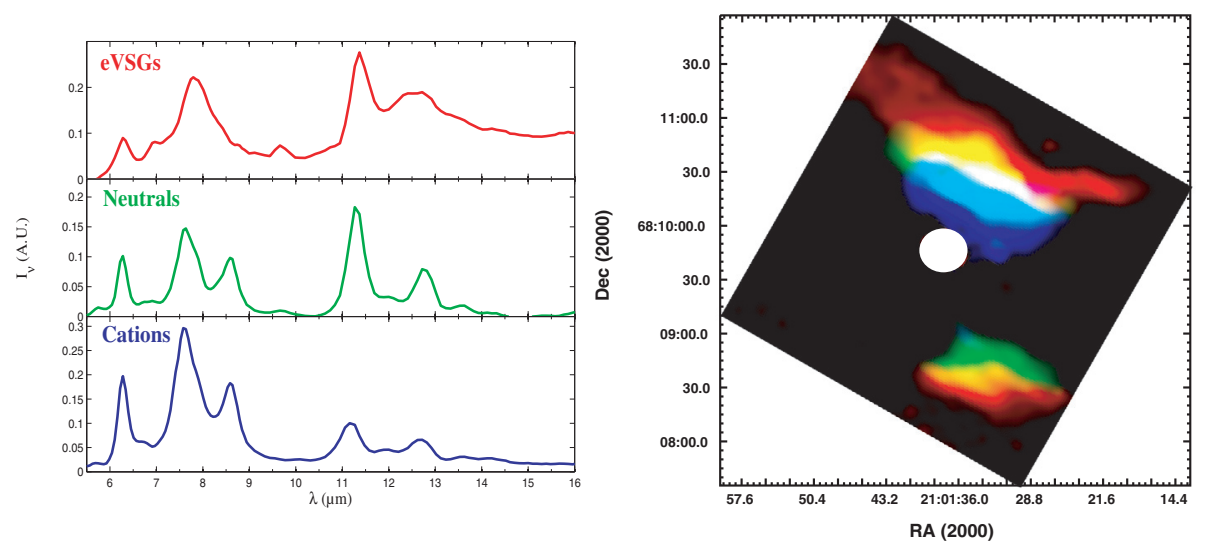

Fig. 1. Left: extracted spectra attributed to evaporating VSGs, $\mathrm{PAH}^{0}$ and $\mathrm{PAH}^{+}$in NGC 7023. Right: associated distribution maps of the three populations: eVSGs in red, $\mathrm{PAH}^{0}$ in green and $\mathrm{PAH}^{+}$in blue. The white circle shows the position of the exciting star. Colors combine as in an RGB image i.e. green $\left(\mathrm{PAH}^{0}\right)+$ red $(\mathrm{eVSGs})=$ yellow.

by physical conditions. This has been well demonstrated on the basis of ISO observations (see e.g. Peeters et al. 2002; Rapacioli et al. 2005) and more recently with Spitzer (see e.g. Werner et al. 2004; Berné et al. 2007). The observed IR spectrum usually consists of a set of bands that are most prominent at $6.2,7.7,8.6,11.3$, and $12.7 \mu \mathrm{m}$. It was established that the modification of the shape of this spectrum can be attributed to the chemical evolution of the emitting populations (Peeters et al. 2002; Hony et al. 2001) as the local physical conditions change. In particular, models (Tielens 2005) and observations (Joblin et al. 1996; Galliano et al. 2008) have shown that the variations of the 6.2 (or 8.6) to $11.3 \mu \mathrm{m}$ band intensity ratio $\left(I_{6.2} / I_{11.3}\right)$ evolves with the ionization parameter $\gamma=G_{0} \times \sqrt{T} / n_{H}$ where $G_{0}$ is the intensity of the UV radiation field in Habing units, $T$ is the gas temperature and $n_{H}$ the total hydrogen nucleus density. Following this work, Berné et al. (2009b) have shown that the combination of the measurement of $I_{6.2} / I_{11.3}$ and of the ratio between the $\mathrm{H}_{2}$ 0-0 S(3) and $\mathrm{S}(2)$ line intensities, respectively at 9.7 and $12.3 \mu \mathrm{m}$, allows to derive the individual values of $T, G_{0}$ and $n_{H}$ when they fall in the ranges $T=250-1500 \mathrm{~K}, n_{H}=10^{4}-10^{6} \mathrm{~cm}^{-3}, G_{0}=10^{3}-10^{5}$ respectively.

\section{Identification of underlying spectral components}

Rapacioli et al. (2005) and Berné et al. (2007) extracted the "pure" spectra of three different populations of PAH-like grains, namely: neutral $\mathrm{PAHs}\left(\mathrm{PAH}^{0}\right)$, ionized PAHs $\left(\mathrm{PAH}^{+}\right)$and evaporating very small grains (eVSGs, see 


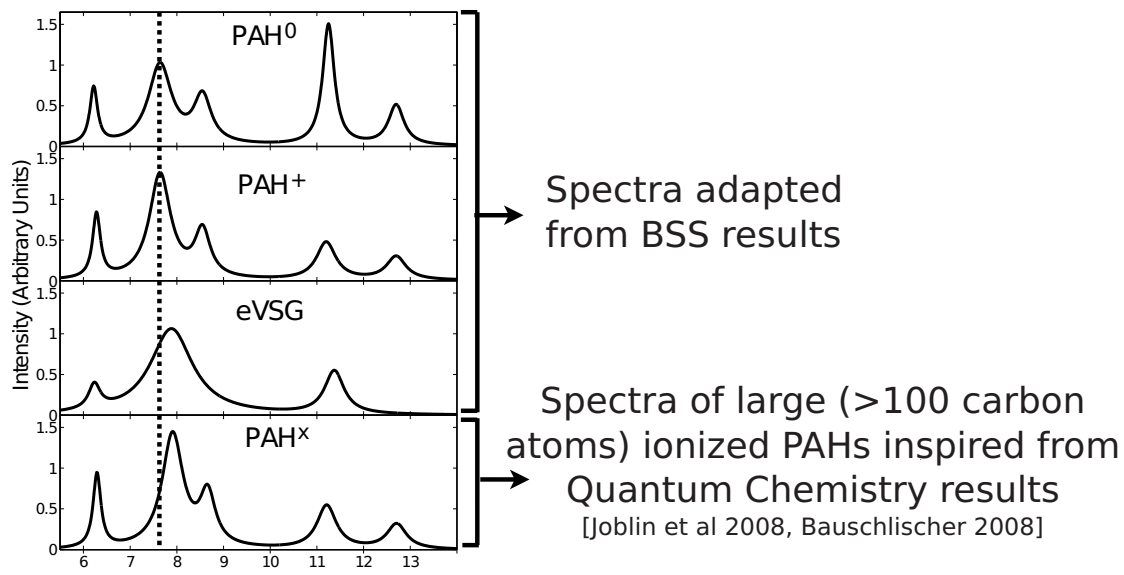

Fig. 2. Template spectra based on the results of Berné et al. (2007) and Rapacioli et al. (2005). In addition, the $\mathrm{PAH}^{x}$ spectrum introduced by Joblin et al. (2008).

Pilleri et al. 2010) in the reflection nebula NGC 7023 using blind signal separation methods. This is interpreted as evidence that the evolution of the shape of the mid-IR bands is related to the chemical evolution under the effect of UV photons (see Fig. 1): free PAH molecules are formed from photoevaporation of very small grains (see Rapacioli et al. in this volume for a review of possible candidates for eVSGs). The main evolution observed for the PAH population is to get ionized closer to the star. Based on these previous results, Joblin et al. (2008) and Berné et al. (2009a), were then able to construct a simple model comprising of eVSG, $\mathrm{PAH}^{0}$ and $\mathrm{PAH}^{+}$mid-IR template spectra (Fig. 2.). While constructing this model, they found that an additional population of PAHs consisting of large (>100 atoms of carbon) and ionized PAHs (called $\mathrm{PAH}^{x}$ ) had to be introduced in order to reproduce the observed spectrum of highly UV-irradiated environments. Other authors (Bauschlisher 2008; Tielens 2008; Geers 2008) have also pointed out the existence of this population of large PAHs.

\section{PAHs/eVSGs: Tracers of physical conditions}

The templates defined in the previous section can be used to fit observed mid-IR spectra of different astrophysical objects such as planetary nebulae (Joblin et al. 2008), protoplanetary disks (Berné et al. 2009a), and galaxies (Vega et al. 2010). One can then relate the results of the fits to the physical conditions as probed by independent tracers, in order to evidence empirical connections. This was done e.g. by Berné et al. (2009a) for protoplanetary disks (Fig. 3). The authors have shown that the fraction of emission attributed to eVSGs in the mid-IR spectra of a sample of 12 disks correlates well with decreasing stellar temperature (Fig. 3). This was interpreted as the more efficient destruction of eVSGs in UV-rich environments. 


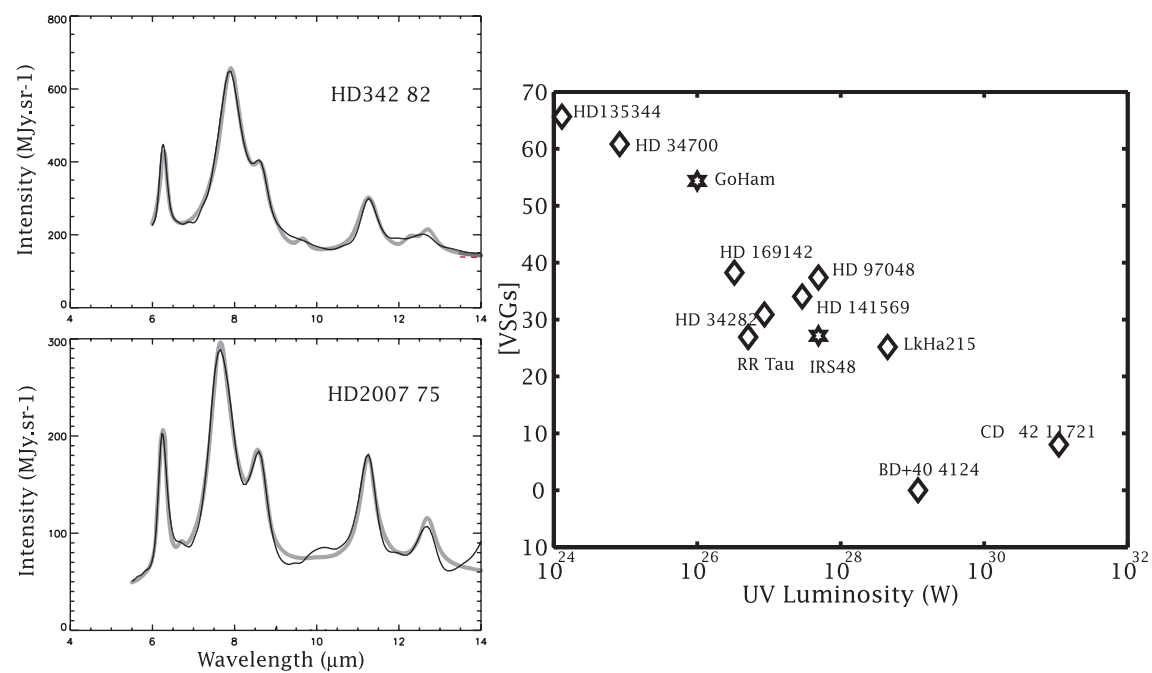

Fig. 3. Left: example of fits (gray), using the template spectra of Figure 2 (corrected for extinction on line of sight), to the observed mid-IR spectra of two protoplanetary disks. Right: the fraction of "evaporating VSG" emission (\%) that was deduced from the fits for a set of 12 observed spectra, as a function of the UV luminosity of the central star (i.e. spectral type).

Recently, a detailed and quantitative analysis comparing the results of PDR models to the results obtained by fitting the set template spectra to mid-IR spectral cubes obtained with Spitzer has shown that, indeed, the eVSG abundance can be used as a tracer of the intensity of the UV field (Pilleri et al. 2010). The template fitting approach can be applied to the mid-IR spectral cubes of nearby galaxies, which in the end provides the spatial distribution of the different populations of PAHs/eVSGs (Fig. 4). This spatial distribution then informs on the local physical conditions of the ISM in different parts of the galaxy. Here, in the case of M 82, the outflow is $\mathrm{PAH}^{0}$-rich, implying the presence of large amounts of molecular gas (as also suggested by Micelotta et al. 2010), shielding the PAHs from ionization and allowing quick recombination of $\mathrm{PAH}^{+}$with electrons. The disk, on the other hand, is filled with $\mathrm{PAH}^{+/ x}$ consistent with the intense star-formation activity producing strong UV fields. Recently, Vega et al. (2010) have applied this fitting approach to the Spitzer spectra of spatially unresolved early type galaxies, and shown the pristine nature of their carbonaceous dust content, implying a constant replenishment of dust by carbon stars. Eventually, one could also use this fitting technique to interpret the mid-IR spectra of galaxies at high redshift and derive their properties. 

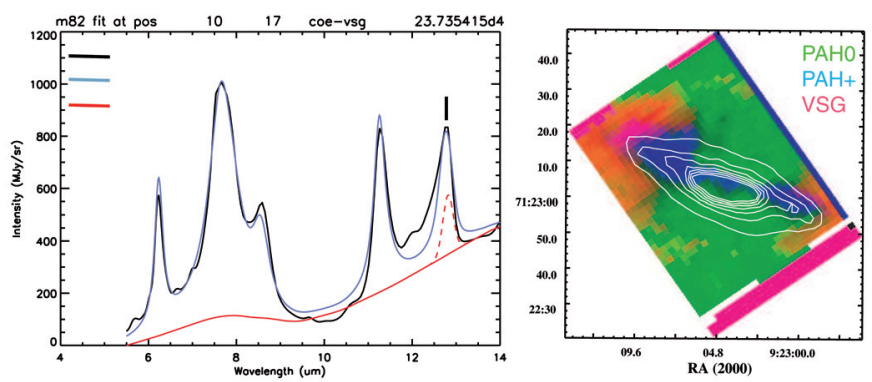

Fig. 4. Example of fit (blue line), using the template spectra of Figure 2 (corrected for extinction on the line of sight), to an observed mid-IR spectrum taken in the cube of M 82 in the outflow region. Achieving the fit on all the spatial positions of the spectral cube, the spatial distribution map of the PAH/eVSG populations can be built (inset). In the inset, white contours show the $3.6 \mu \mathrm{m}$-mostly- stellar emission.
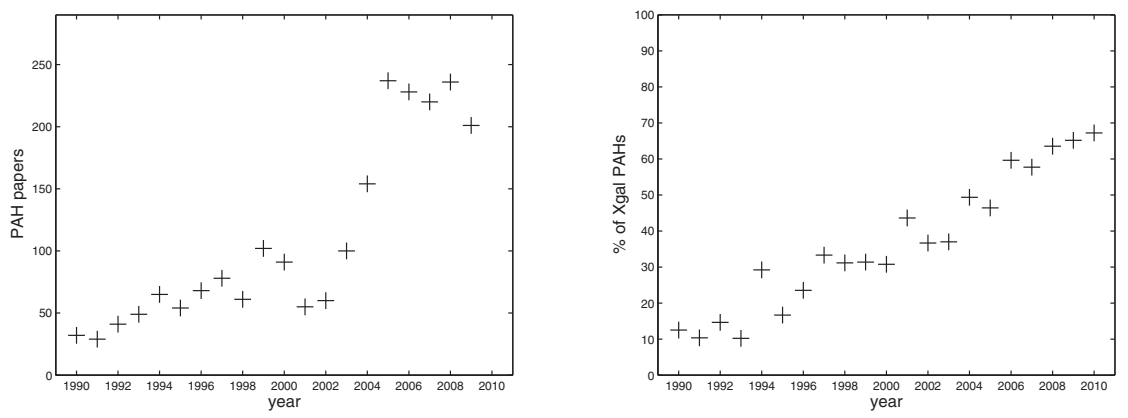

Fig. 5. Left: number of published papers each year regarding PAHs in astronomy in the last 20 years. Right: percentage of papers regarding PAHs that focus on PAHs observed in external galaxies in the last 20 years. Source: NASA-ADS.

\section{Perspectives in astronomical PAH science}

The improvement of ground- and space-based instruments in the mid-IR have provided us with an incredible legacy of astronomical PAH spectra. This has, in the last 20 years, motivated each year more studies (Fig. 5). As we have briefly illustrated in the previous section, the PAH spectrum contains a wealth of information on the physical conditions of the emitting source. More and more, these bands are used as a tracer of physical conditions in UV-irradiated astrophysical environments (protoplanetary disks, galaxies, ... ) because they are easy to detect (Fig. 5). This is why knowing the detailed composition of PAHs, their evolution and chemistry in space remains one of the key challenges of modern astrophysics. The ISO and Spitzer legacies, the Herschel surveys now (see Joblin et al. in this 
volume), and the SPICA (Berné et al. 2009c), JWST and ELT observations in the future, will contribute to this task.

\section{References}

Berné, O., Joblin, C., Mulas, G., Tielens, A.G.G.M., \& Goicoechea, J.R., 2009c, SPICA joint European/Japanese Workshop, July, 2009

Berné, O., Fuente, A., Goicoechea, J.R., et al., 2009b, ApJL, 706, 160

Berné, O., Joblin, C., Fuente, A., \& Ménard, F., 2009a, A\&A, 495, 827

Berné, O., Joblin, C., Deville, Y., et al., 2007, A\&A, 469, 575

Galliano, F., Madden, S.C., Tielens, A.G.G.M., Peeters, E., \& Jones, A.P., 2008, ApJ, 679,310

Hony, S., Van Kerckhoven, C., Peeters, E., et al., 2001, A\&A, 370, 1030

Joblin, C., Szczerba, R., Berné, O., Szyszka, C., et al., 2008, A\&A, 490, 189

Joblin, C., Tielens, A.G.G.M., Geballe, T.R., \& Wooden, D.H. 1996, ApJ, 460, L119

Peeters, E., Hony, S., Van Kerckhoven, C., et al., 2002, A\&A, 390, 189

Rapacioli, M., Joblin, C., \& Boissel, P., 2005, A\&A, 429, 193

Micelotta, E.R., Jones, A.P., \& Tielens, A.G.G.M., 2010, A\&A, 510, 37

Tielens, 2008, ARA\&A, 46, 289

Tielens, A., 2005 (Cambridge University Press)

Vega, O., Bressan, A., Panuzzo, P., et al., 2010, ApJ, 721, 1090

Werner, M.W., Uchida, K.I., Sellgren, K., et al., 2004, ApJS, 154, 309 